\title{
TABAK, IBRIK, FINCAN ET AUTRES POTS D'ÉPOQUE OTTOMANE AU BILÂD AL-CHÂM
}

'étude de la vaisselle fabriquée et commercialisée dans l'Empire ottoman est un domaine encore peu exploité de la discipline céramologique. Des travaux épars et de natures diverses, écrits tant par des historiens de l'art que par des archéologues, ont livré des indications de toutes natures sur ce matériel. Cependant, les typologies de référence permettant de distinguer, par périodes chronologiques et par centres de fabrication, les céramiques qui répondent aux nécessités domestiques habituelles, que ce soit la vaisselle culinaire, de service et de table ou les céramiques de stockage et de transport, sont encore très rares comme le montrera l'inventaire des principales découvertes faites sur les territoires de la Sublime Porte. Dans les provinces arabes de l'empire, ce sont incontestablement les céramiques mises au jour au Bilâd al-Châm qui sont les mieux connues, même si là aussi il reste beaucoup à faire pour en dresser un tableau complet et bien daté. Dans un tel contexte, la fouille récente de la citadelle de Damas offre une opportunité remarquable d'améliorer notre connaissance de la vaisselle en usage au Proche-Orient du $\mathrm{XVI}^{\mathrm{e}}$ au début du $\mathrm{XX}^{\mathrm{e}}$ siècle. L'étude de ces objets de terre, combinée à celle des sources écrites, livrera une image plus vivante des intérieurs damascènes et des pratiques de table en cours à ces époques tardives. À travers quelques exemples, il sera aussi montré combien le croisement des sources écrites et des artefacts peut être fructueux car il est

Véronique FrANçoIs est chargée de recherche au CNRS, Institut français du Proche Orient, Damas (Syrie).

francois@scs-net.org 
important de dépasser les problèmes habituels de chronologie et d'attribution pour aborder la fonction des objets et leur utilisation. Au-delà des bilans des découvertes de céramiques ottomanes et des études qui leur sont consacrées, cet article se propose aussi de mettre en lumière de nouvelles pistes de recherches et ainsi, peut-être, de contribuer au développement d'un champ de la céramologie peu traité.

\section{BILAN DES RÉSULTATS CONNUS SUR LA CÉRAMIQUE}

\section{DANS L'EMPIRE OTTOMAN}

Longtemps, les publications traitant de vaisselle d'époque ottomane ont été exclusivement consacrées aux plus fameuses productions de l'Empire, c'est-à-dire: aux céramiques à pâte siliceuse très dure, peintes polychromes, qui firent la renommée des ateliers anatoliens d'Iznik de la fin du XVe à la fin du XVII ${ }^{\mathrm{e}}$ siècle - le superbe volume de J. Raby et N. Atasoy en étant vraisemblablement la publication la plus achevée ${ }^{1}$; aux céramiques siliceuses de Kütahya, fabriquées au XVIII ${ }^{\mathrm{e}}$ siècle en Anatolie centrale ${ }^{2}$; et aux productions de vaisselle à pâte argileuse des ateliers de Çanakkale implantés sur la rive asiatique du détroit des Dardanelles, en activité du début du $\mathrm{XVIII}^{\mathrm{e}}$ au début du $\mathrm{XX}^{\mathrm{e}}$ siècle ${ }^{3}$. Cette vaisselle fine, étudiée du point de vue de l'histoire de l'art est issue, pour l'essentiel, de collections privées et publiques ${ }^{4}$. Rares sont les travaux présentant aussi les fabrications qui constituent la plus grande part de la production courante, c'est-à-dire une vaisselle culinaire, de service et de table, des céramiques de stockage et de transport ainsi que des luminaires. Cet aspect des productions est moins connu, bien que le matériel existe comme en attestent les découvertes archéologiques. Un tour d'horizon de ces trouvailles et de la manière dont elles ont été traitées permet d'en évaluer l'ampleur et la diversité.

${ }^{1}$ N. Atasoy, J. Raby, Iznik, La poterie en Turquie ottomane, Ed. du Chêne, 1990.

2 J. Carswell, Kütahya Tiles and Pottery from the Armenian Cathedral of St. James, vol. II, Oxford, 1972; idem, «A Minor Group of Late Turkish Pottery», Sanat Tarihi Yllığl, I, 1964-1965, p. 1-15.

${ }^{3}$ G. ÖNEY, Türk devri Çanakkale Seramikleri-Turkish Period Çanakkale Ceramics, Ankara, 1971.

${ }^{4}$ Voir par exemple le beau catalogue consacré aux collections du Suna-Inan Kıraç et du musée Sadberk Hanım, L. SousTiEL, Splendeurs de la céramique ottomane du XVI ${ }^{e}$ au XIXe siècle, Istanbul, 2000. 
En Turquie, la céramique d'époque ottomane provient des fouilles de sites antiques ou byzantins réoccupés durant la période moderne. Mais le plus souvent, ces objets, recueillis en dehors de tout contexte stratigraphique, sont privés de datation fiable. Ainsi à Iznik, la mise en évidence d'une production de céramique à pâte rouge et à glaçure plombifère, de facture assez grossière, a révélé l'existence, aux côtés de la vaisselle de luxe, d'une fabrication locale d'objets usuels de moindre qualité destinés à répondre aux besoins quotidiens de la population. Mais les contextes de découverte de ce matériel - divers travaux de creusement effectués dans la ville et fouilles du théâtre romain - ne fournissent aucune indication chronologique sûre ${ }^{5}$. À Aphrodisias de Carie, le bel ensemble constitué de céramiques culinaires, de vases de stockage avec ou sans revêtement et de vaisselle de service et de table glaçurée de facture assez rustique, sans doute produit localement ou régionalement, est daté entre le $\mathrm{XV}^{\mathrm{e}}$ et le $\mathrm{XIX}^{\mathrm{e}}$ siècle sur la base d'analogies et grâce aux importations turques et européennes ${ }^{6}$. Pour leur part, les fouilles de Sardes en Lydie ${ }^{7}$ ont livré des poteries - considérées comme des productions locales - qui proviennent de niveaux turcs très perturbés, datés entre le début du XIV e et le XVIII ${ }^{\mathrm{e}}$ siècle. Si l'identification et la datation des céramiques fines telles que la vaisselle d'Iznik, les céramiques dites de Milet, celles de Kütahya et les porcelaines de Chine ne posent pas de problème majeur, il n'en est pas de même pour toutes les céramiques à pâte rouge, incisées ou non, à glaçure monochrome ou polychrome trouvées sur le site. Enfin à Ephèse, des pernettes et des rebuts de cuisson témoignent, cette fois assurément, d'une fabrication locale entre le $\mathrm{XIII} \mathrm{I}^{\mathrm{e}}$ et le $\mathrm{XV}^{\mathrm{e}}$ siècle — les résultats de l'étude actuellement menée par J. Vroom sur ce matériel sont très attendus. À Istanbul, si les informations concernant la vaisselle ottomane livrées par les fouilles anciennes du Grand Palais ${ }^{8}$, ou celles plus récentes

5 V. FrançOIS, «Céramiques ottomanes de tradition byzantine d'Iznik », AnatAnt, IV, 1996, p. 231-245; N. Özkul FiND1K, Iznik Roma Tiyatrosuyu Kazı Buluntularl (19801995) Arasındaki. Osmanl Seramikleri, Ankara, 2001, p. 85-169.

6 V. FrançoIs, «Eléments pour 1'histoire ottomane d'Aphrodisias: la vaisselle de terre », AnatAnt, IX, 2001, p. 147-190.

7 J.A. ScotT, D.C. Kamilli, «Late Byzantine Glazed Pottery from Sardis», $X V^{e}$ ACIEB, Athènes, septembre 1976, II, 2, Athènes, 1981, p. 686; H.G. CRANE, «Preliminary Observations on the Glazed Pottery of the Turkish Period from Sardis », BASOR, 228, 1977, p. 51-54; idem, «Some Archaeological Notes on Turkish Sardis », Muqarnas, 4, 1987, p. 44-58.

8 R.B.K. Stevenson, The Pottery, The Great Palace of Byzantine Emperors, First Report 1935-1938, Londres, 1949, p. 60. 
de Kalenderhane Camii ${ }^{9}$, sont difficilement utilisables que ce soit d'un point de vue typologique ou chronologique, les découvertes de Bodrum Camii ${ }^{10}$ et de Saraçhane Camii ${ }^{11}$ sont essentielles car le matériel y apparaît en contexte stratigraphique. Au Myrélaion (Bodrum Camii), la plus grande partie de la vaisselle, qui comprend des céramiques fines turques et importées ainsi que divers types de poterie domestique locale, date de la première moitié du $\mathrm{XVI}^{\mathrm{e}}$ siècle tandis qu'une plus petite quantité est attribuée au XVIII ${ }^{\mathrm{e}}$. Les nombreux fragments ottomans recueillis à Saraçhane Camii fournissent un échantillonnage des types les plus courants de 1500 à 1650 , la période s'étendant de 1700 à 1850 étant plus faiblement représentée. Au contraire de la pratique habituelle, J.W. Hayes consacre une place respectable aux céramiques communes sans revêtement ou glaçurées. Ces découvertes stambouliotes, importantes, constituent des lots de référence pour toute la période et pour tous les territoires de l'Empire.

Dans les Balkans, la vaisselle ottomane a jusqu'alors suscité assez peu d'intérêt, sans doute parce qu'elle renvoie à une période sombre souvent désignée par les termes de «Turcocratie» ou de «joug ottoman». Les conséquences de cette «occupation» sont encore trop présentes dans les esprits pour qu'une quelconque attention ait été portée aux vestiges qui subsistent encore. Ainsi en Grèce, les découvertes de vaisselle ottomane semblent assez rares, pour le moins elles sont peu publiées et qualifiées non pas d'ottomanes mais de post-byzantines. Les précurseurs des recherches en ce domaine furent d'abord des étrangers. A. Frantz publie, dès 1942, le matériel d'époque ottomane mis au jour dans les fouilles américaines de l'Agora d'Athènes, qui constitue à ce jour l'ensemble le plus considérable trouvé en Grèce ${ }^{12}$. À l'occasion de ces fouilles, des fours, des pernettes et des ratés de cuisson, découverts près de la Stoa Sud et sous l'église de Varassou, témoignent d'une production athénienne dont le terminus post quem correspond à 1676. La même année, dans son volume consacré à l'abondante vaisselle byzantine de Corinthe, C. Morgan, dans un petit chapitre, traite aussi des céramiques post-

9 A. SABuncu, «A Note on Ceramics from Kalenderhane Camii», DOP, 29, 1975, p. 316.

10 J.W. HAYES, «The Excavated Pottery from the Bodrum Camii», in C.L. Striker, The Myrelaion (Bodrum Camii) in Istanbul, Princeton University Press, 1981, p. 36-47.

11 J.W. HAYES, Excavations at Saraçhane in Istanbul, volume II : the Pottery, Princeton, 1992.

12 A. FrantZ, «Turkish Pottery from the Agora», Hesperia, 11, 1, Athènes, 1942, p. $1-28$. 
byzantines du $\operatorname{site}^{13}$. Quelques décennies plus tard, J. Carswell dresse un inventaire des bacini d'époque ottomane des monastères du Mont Athos ${ }^{14}$. Il s'agit essentiellement de vaisselle fine d'Iznik et de Kütahya et d'importations iraniennes, européennes et chinoises. Dans les années 1980-90, la plus grande part des articles consacrés aux productions postbyzantines a été rédigée par $\mathrm{A}$. Charitonidou qui confirme notamment l'existence d'ateliers à Athènes en fonctionnement du milieu du XVII jusqu'au milieu du XIX ${ }^{\mathrm{e}}$ siècle $^{15}$. Aujourd'hui, un intérêt nouveau semble se manifester pour ces productions tardives comme le révèle le volume consacré à la céramique byzantine à glaçure, édité, en 1999, à l'occasion du $\mathrm{VII}^{\mathrm{e}}$ Congrès international sur la céramique médiévale en Méditerranée. On y trouve en effet plusieurs notices bien illustrées traitant d'ateliers de céramiques à glaçure post-byzantines mis au jour en Grèce du Nord, à Thessalonique, à Véria et à Trikala ${ }^{16}$. Ces vases sont attribués, sur des bases un peu floues, aux $\mathrm{XV}^{\mathrm{e}}-\mathrm{XVI}^{\mathrm{e}}$ siècles et aux $\mathrm{XVI}^{\mathrm{e}}-\mathrm{XVII}{ }^{\mathrm{e}}$ siècles. L'existence d'un autre centre en Thrace, très important du point de vue de la diffusion de ses productions, avait déjà été révélée par Ch. Bakirtzis qui présentait, en 1980, les ateliers de Didymotique, une ville implantée au bord de l'Evros et un grand centre de fabrication potière du $\mathrm{XIV}^{\mathrm{e}}$ au $\mathrm{XX}^{\mathrm{e}}$ siècle $^{17}$ — selon Evliya Çelebi, au $\mathrm{XVII}^{\mathrm{e}}$ siècle, la ville compte en effet deux cents ateliers de potier. C'est aussi à $\mathrm{Ch}$. Bakirtzis que nous devons la publication du four post-byzantin de Ferrès dans la région de $1^{\prime}$ Evros ${ }^{18}$. Il semble en effet qu'en Thrace

13 C. Morgan, Excavations at Corinth XI: the Byzantine Pottery, Cambridge Mass., 1942, p. 171-180.

14 J. Carswell, «Pottery and Tiles on Mount Athos», Ars Orientalis, VI, 1966, p. 77-90.

15 A. Charitonidou, «Céramique grecque du XVII ${ }^{\mathrm{e}}$ siècle dans la ville d'Arta », Faenza, 66, 1980, p. 375-377; eadem, «Morphes metabyzantinis keramikis, 'Athenaika ergasteria», Archailologia, 4, 1982, p. 60-64; A. VAVYLOPOUlOU-CHARITONIDOU, «Metabyzantina aggeia apo to kastro ton Rogon », Epeirotika Chronika, 28, 1986/87, p. 37-42; eadem, «Céramiques d'offrande trouvées dans des tombes byzantines tardives de 1'Hippodrome de Thessalonique », in V. Déroche, J.M. Spieser (eds.), Recherches sur la céramique byzantine, BCH Supplément XVIII, Athènes, 1989, p. 209-232.

16 D. PAPANIKola-BAKIRTZIS, "Thessaloniki Workshop Production», in D. Papanikola-Bakirtzis (ed.), Byzantine Glazed Pottery, Athènes, 1999, p. 249-253; G. PAPAzOTOU, «Veria Workshop Production», ibid., p. 254-257; K. MantZanA-SaPOunA, «Trikala Workshop Production», ibid., p. 258-265.

17 C. BAKIRTZIS, «Didymoteichon: un centre de céramique post-byzantine», BalkStud, 21, 1, 1980, p. 147-153; K. TsourIs, «Ektesi anaskaphikis ereunas sto Didymoticho », $A A A, 20,1987$, p. 43-65.

18 Ch. BAKIRTZIS, «Fouille d'un four post-byzantin à Pherrès », ArchDelt, 32 II, 1977, p. 283-284, pl. 165-167. 
et en Macédoine, la production de vaisselle de terre à l'époque ottomane était assez développée comme le signale encore Evliya Çelebi qui mentionne l'existence d'ateliers à Ainos (Enez), Selânik, Midye et Dimoteka (Didymoteichon) ${ }^{19}$. En Crète et à Chypre, la présence de vaisselle postbyzantine est aussi attestée sur les fouilles, elle est l'objet d'articles qui mettent en évidence les importations et les productions locales d'un point de vue archéologique ${ }^{20}$. Un intérêt encore timide pour les poteries post-médiévales se manifeste ailleurs dans les Balkans comme en témoignent l'ouvrage récent consacré au matériel des $\mathrm{XVI}^{\mathrm{e}}-\mathrm{XVII}{ }^{\mathrm{e}}$ siècles découvert dans la forteresse de Belgrade ${ }^{21}$ et l'étude en cours dédiée aux céramiques ottomanes de Bulgarie ${ }^{22}$.

L'histoire des terres cuites communes et à glaçure fabriquées et commercialisées dans les provinces arabes de l'Empire ottoman reste à écrire. Au Bilâd-al-Châm, comme nous allons le voir, les découvertes archéologiques concernant cette période sont assez nombreuses et suscitent un intérêt nouveau alors qu'en Egypte, ce matériel est quasiment inconnu. On n'en trouve mention que de façon épisodique et seules les importations sont signalées faute de fouilles permettant de distinguer les vases d'usage courant produits et utilisés du début du $\mathrm{XVI}^{\mathrm{e}}$ au XVIII siècle $^{23}$. Si on ne sait rien des pièces de formes, en revanche, quelques travaux ont été consacrés aux carreaux ottomans qui ornent diverses mosquées du Caire ${ }^{24}$. À Tunis, conquise par Sinan Pacha en 1574, une

19 J. von HAMmer (trad.), Narratives of Travel in Europe, Asia and Africa in the Seventeenth Century by Evliyá Efendi, I, 2, Londres, 1834-1846, p. 31-32.

20 T. Stillwell MacKay, «A Group of Renaissance Pottery from Heraklion, Crete: Notes and Questions », in P. Lock, G.D.R. Sanders, The Archaeology of Medieal Greece, Oxford, 1996, p. 127-138; M. HAHN, «Filterjugs and Lampstands from the Turkish Period found at Khania » in Proceedings of the 6th International Cretological Congress at Khania 1986, La Canée, 1990, p. 77-82 ; eadem, «Modern Greek, Turkish and Venetian Periods », in E. et B.P. Hallager (eds.), The Greek-Sweedish Excavations at the Agia Aikaterini Square, Kastelli, Khania 1970-1987, vol. I, 1, Stockholm, 1997, p. 79-192; M.L. Von Wartburg, "Types of Imported Table Ware at Kouklia in the Ottoman Period», RDAC, 2001, p. 361-396; V. FRANÇOIS, L. VALLAURI, «Production et consommation de céramiques à Potamia (Chypre) de l'époque franque à l'époque ottomane », BCH, 125, 2, 2001, p. 523-546.

${ }^{21}$ V. BIKIC, Gradska keramika Beograda (16-17.vek) - Belgrade Ceramics in the $16^{\text {th }}-17^{\text {th }}$ Century, Belgrade, 2003.

22 Thèse de doctorat de G. Guionova au LAMM d'Aix-en-Provence.

23 R.P. Gayraud, C. DÉcobert, «Fouilles islamiques d'Al-Qasaba (oasis de Dakhla) - $1980 »$, AnIsl, 18, 1982, p. 285, note 1; R.P. GAYRAUD, «La céramique des fouilles d'Al-Qasaba (oasis de Dakhla)», AnIsl, 20, 1984, p. 145; V. FrançOIs, Céramiques médiévales à Alexandrie, Etudes Alexandrines 2, Le Caire, 1999, p. 133-138 et 176-177.

24 V. MeineCKe-Berg, «Osmanische Fliesendekorationen des 17. Jahrhunderts in 
forte influence décorative ottomane apparaît en particulier dans l'ornementation des piédroits et sur les linteaux des portes ainsi que sur les plafonds de bois peints et sur les panneaux de faïence murale, des supports sur lesquels on observe divers éléments floraux turquisants sculptés ou peints ${ }^{25}$. Les maîtres céramistes des ateliers de Qallaline - des officines situées dans les faubourgs immédiats de Tunis entre Bab Souika et Bab el-Jazira - ne manquent pas de s'inspirer des thèmes décoratifs ottomans plus ou moins gauchement interprétés pour réaliser des panneaux de style turc, connus sous le nom de «torki», qui puisent au répertoire décoratif anatolien les motifs floraux typiques comme les œillets, les tulipes, les palmettes, les roses ou la feuille $s a z$; les médaillons flammés avec des bouquets de fleurs peints en réserve; les médaillons turco-persans, les palmes persanes et le décor dit à $m i h r a b^{26}$. Cependant si une partie de la production de carreaux est incontestablement marquée par ces nouveaux éléments décoratifs venus d'Anatolie, la production de vaisselle de Qallaline semble assez peu concernée par la céramique turque dont elle ne s'inspire ni dans le décor - à de rares exceptions près ${ }^{27}-$ ni dans les formes des vases. Pour cette période, en Tunisie, seule la vaisselle de table est connue et rien n'est publié sur les céramiques communes.

Il est encore délicat de tenter des mises en perspectives de ces différents ensembles, les données sont trop disparates pour distinguer des différences régionales ou, au contraire, souligner des permanences dans les formes ou dans les décors quelles que soient les provinces de l'Empire. De la même façon, il est encore trop tôt pour essayer de dresser un tableau comparatif des modes d'approvisionnement en vaisselle étrangère souvent présente sur les sites ottomans en petites quantités. Compte tenu de l'état encore assez lacunaire de nos connaissances sur ce matériel,

Kairo », in IV Congrès International d'art turc, Aix-en-Provence, 10-15 septembre 1971, Aix-en-Provence, 1976, p. 155-159; Ü. Ü. BATES, «Evolution of Tile Revetments in Ottoman Cairo », First International Congress of Turkish Tiles and Ceramics, Kütahya, 611 July 1986, Istanbul, 1989, p. 39-58.

25 J. Revault, Palais et demeures de Tunis (XVIII et XIX e siècles), Paris, 1971 ; idem, «Influence de l'art turc sur l'art tunisien entre la fin du XVI ${ }^{\mathrm{e}}$ et le XIX ${ }^{\mathrm{e}}$ siècle », IVe Congrès International d'art turc, Aix-en-Provence, 10-15 septembre 1971, Aix-en-Provence, 1976, p. 201-204 ; A. SAADAOUI, Tunis, ville ottomane, Tunis, 2001.

26 A. Loviconi, D. Belfitah, Faïences de Tunisie, Qallaline et Nabeul, Aix-en-Provence, 1994; A. LouHICHI, «La céramique de Qallaline» in Couleurs de Tunisie, Institut du monde arabe, Paris, 13 décembre 1994-26 mars 1995, Paris, 1994, p. 183-253.

27 A. LouHICHI, 1994, op. cit., p. 194, 195. 
toute nouvelle découverte d'importance, où qu'elle soit faite dans l'Empire ottoman, contribue à une meilleur définition des objets de terre cuite fabriqués et commercialisés à cette époque tardive. L'étude des céramiques mises au jour dans les fouilles de la citadelle de Damas entre 1999 et 2003 fournira des indications nouvelles à l'échelle régionale ainsi qu'à l'échelle de l'Empire tout entier. Mais avant d'examiner ce matériel, il n'est pas inutile de brosser un tableau des découvertes faites du Bilâd al-Châm pour montrer que les lots de références dont on dispose sont assez peu nombreux.

CÉRAMIQUES D'ÉPOQUE OTTOMANE AU BILÂD AL-CHÂM :

UNE PRÉSENCE ATTESTÉE MAIS ENCORE TROP RAREMENT EXPLOITÉE

En Syrie, au Liban, en Jordanie et en Israël, une activité archéologique très dynamique explique l'abondance des découvertes céramologiques qui, cependant, n'ont pas toutes bénéficié du même traitement selon les époques qu'elles illustrent. Sur ce vaste territoire qu'est le Bilâd al-Châm, il existe en effet une forte distinction dans la spécialisation en fonction du découpage par dynasties. M.-O. Rousset, qui propose un tableau partiel des trouvailles de céramiques des $\mathrm{XI}^{\mathrm{e}}$ et $\mathrm{XII}^{\mathrm{e}}$ siècles au Proche-Orient, montre que la période abbasside est bien documentée et que les nombreuses découvertes de la période islamique moyenne ont bénéficié de l'intérêt porté avant tout à la présence croisée au Levant ${ }^{28}$. Dans l'ensemble, les sites du XII ${ }^{\mathrm{e}}$ siècle sont mieux connus que ceux du $\mathrm{XI}^{\mathrm{e}}$. Il semble par ailleurs que les productions d'époque ayyoubide ont un peu éclipsé les trouvailles mameloukes comme le souligne D. Whitcomb dans le bilan qu'il dresse des études archéologiques traitant de cette époque ${ }^{29}$. Il note cependant que cet eurocentrisme, lié à l'attention portée aux croisades, a tendance à diminuer. Dans ce contexte, la vaisselle utilisée et commercialisée au Bilâd al-Châm entre 1516 et 1918 est mal connue alors que les données matérielles existent comme le révèle un inventaire très complet des sites du Proche-Orient

${ }^{28}$ M.-O. Rousset, «La céramique des $\mathrm{XI}^{\mathrm{e}}$ et $\mathrm{XII}^{\mathrm{e}}$ siècles en Egypte et au Bilād alShām. État de la question », in M. Barrucand (dir.), L'Egypte fatimide, son art et son histoire, PU Paris-Sorbonne, p. 250-264.

${ }_{29}^{2}$ D. Whiтcомв, «Mamluk Archaeological Studies: A Review», Mamluk Studies Review, 1, 1997, p. 97-106. 
ayant livré des vases d'époque ottomane ${ }^{30}$. C'est de cet inventaire, établi par M. Milwrigt en 2000, que sont extraits les tableaux, reproduits en annexe à la fin de cet article, qui donnent le détail des découvertes apparemment nombreuses mais dont il est difficile d'évaluer le volume. Paradoxalement, alors que la présence de vaisselle de terre ottomane est souvent signalée dans les publications, elle est rarement étudiée et, même dans ce cas, peu illustrée. De façon générale, la céramique de ces époques tardives est peu considérée et ceci pour plusieurs raisons. Elle souffre d'abord de sa «modernité», les niveaux ottomans, les plus récents dans la succession des strates, plus sensibles aux perturbations contemporaines, sont souvent moins bien préservés et les fouilles ayant fourni des datations fiables sont rares. Cette insuffisance de données sûres au niveau chronologique rend difficile l'élaboration de typologies de référence. Par ailleurs, il est souvent avancé, pour justifier ce manque de classification, une permanence des types entre l'époque mamelouke tardive et le début de l'époque ottomane. Cet argument n'est pas recevable comme le démontre le matériel d'Acre ou de Damas - mais nous y reviendrons. L'identification des trouvailles ponctuelles est également rendue difficile par la taille du territoire considéré dans lequel il existe des variantes régionales. Ainsi la typologie établie pour Acre ne sera pas forcément utile pour traiter du matériel mis au jour sur tels sites de Jordanie ou de Syrie ${ }^{31}$. Enfin les contextes de découvertes sont de diverses natures, il s'agit de ports, de fundunq-s, de forteresses rurales et d'habitations urbaines ou villageoises. Les assemblages peuvent donc être très variables en fonction de l'aire géographique, d'une part, et de la nature du site fouillé, d'autre part. Malgré tous ces obstacles, quelques découvertes encore peu abondantes, faites dans des contextes calés chronologiquement, fournissent des indications précises sur d'assez courtes périodes, comme le montre $\mathrm{M}$. Milwright dans la première partie de son article $^{32}$. Ces résultats, ajoutés les uns aux autres, contribueront peu à

${ }^{30}$ M. Milwright, «Pottery of Bilad al-Sham in the Ottoman Period: a Review of the Published Archaeological Evidence », Levant, 32, 2000, p. 189-208.

31 À St Jean d'Acre, la fouille d'un dépôt clos, un puits dans une tour croisée a livré une collection de vases dont la fonction est étroitement liée au puits lui-même — des godets de noria pour puiser l'eau, des jarres pour la transporter, des cruches pour la conserver et la servir. Une partie de ces objets est réalisée avec la même pâte que celle utilisée pour fabriquer les Acre Bowls d'époque croisée. Ce qui implique une fabrication locale pour ces céramiques communes datées, grâce à la stratigraphie et au contexte historique, du XVIII' siècle. E. STERn, «Excavation of the Courthouse Site at 'Akko: The Pottery of the Crusader and Ottoman Periods », 'Atiqot, XXXI, 1997, p. 35-70.

32 M. Milwright, 2000, op. cit., p. 191-193. 
peu au développement de classifications plus sûres. Dans ce contexte, l'importante quantité de céramiques ottomanes recueillie dans les récentes fouilles ouvertes à la citadelle de Damas, offre une opportunité exceptionnelle de traiter de la vaisselle en usage dans un grand site urbain entre 1516 et 1918 .

LA VAISSELLE DE TERRE EN USAGE À LA

CITADELLE DE DAMAS DE 1516 à 1918 :

DES DONNÉES NOUVELLES EN COURS D'EXPLOITATION

À la demande de la Direction générale des Antiquités et des Musées de Syrie, un programme archéologique franco-syrien s'est mis en place, en 1999, à la citadelle de Damas ${ }^{33}$. Dix-huit mois de fouilles ouvertes principalement dans une vaste salle d'apparat et dans un grand bâtiment de quatre niveaux ont livré un important matériel qui couvre les époques seldjoukide, ayyoubide et mamelouke ainsi que la totalité de l'époque ottomane. Pour cette dernière, on dénombre 2235 fragments de bords ou de bases et 6075 fragments de panses. Le volume et la variété des céramiques trouvées au cours de ces fouilles permettront d'établir une typologie de la vaisselle de terre en usage dans cette «ville-garnison» de 1516 à 1918.

L'étude de ces vestiges de terre cuite est en cours mais il est possible de présenter quelques résultats préliminaires

\section{Damas, un centre de production à l'époque ottomane}

Il semble que la production de vaisselle à Damas, établie pour les périodes ayyoubide et mamelouke notamment dans le quartier de Bāb Charqī, se soit poursuivie à l'époque ottomane au même endroit. Sur la carte de Damas, dressée à partir des textes par J. Sauvaget pour le début du XVI ${ }^{\mathrm{e}}$ siècle, des ateliers de potiers sont localisés à Salihyé et dans le quartier israélite comme à l'époque mamelouke mais aussi à l'Est des murailles, en face de Bāb Charqi $\overline{1}^{34}$. Si on constate une certaine perma-

33 Cette fouille est dirigée par S. Berthier (IFPO, Damas) et E. al-Aji (DGAMS). Le dossier sur la céramique des époques seldjoukide et ayyoubide a été confié à St. McPhillips (doctorant de l'université de Sydney); quant à moi, j'ai en charge les périodes mamelouke et ottomane. L'étude est en cours.

34 J. Sauvaget, «Esquisse d'une histoire de la ville de Damas », REI, VIII, 1934. 
nence dans l'implantation des ateliers, de l'époque ayyoubide à l'époque ottomane, les potiers semblent avoir déserté cependant le sud-ouest de la ville et l'extrémité occidentale de la rue Droite. Des vases surcuits mis au jour à la citadelle témoignent bien d'une production locale. Damas est par ailleurs bien connu comme centre producteur de copies de céramiques et de carreaux d'Iznik comme le confirment des analyses physicochimiques des pâtes ${ }^{35}$. Étant donné le peu d'analogie existant entre le matériel de Damas et les autres trouvailles faites au Proche-Orient, il est permis de supposer que la plupart des céramiques de la citadelle ont été réalisées localement. En l'absence de découverte de fours ou de dépotoirs, des vestiges de l'activité potière ottomane, cela reste bien entendu une hypothèse.

\section{Résultats préliminaires sur la céramique commune et la vaisselle fine «locale»}

Les céramiques communes, pour l'ensemble de la période, se répartissent de façon quasiment égale en pâte rouge et en pâte grise, on ne trouve plus aucune trace de productions à pâte argileuse claire fréquentes dans les niveaux ayyoubides et mamelouks. J.W. Hayes constatait, à partir du matériel de Saraçhane, que les céramiques à glaçure plombifère s'étaient substituées à la vaisselle culinaire à paroi fine ${ }^{36}$. Un phénomène semblable s'observe à Damas. À la différence des céramiques communes mameloukes, les jarres, les bassins, les coupes et les jattes ainsi que les vases à eau ottomans sont majoritairement glaçurés aux $\mathrm{XVI}^{\mathrm{e}}$ et $\mathrm{XVII}^{\mathrm{e}}$ siècles. Plus tard, les potiers recourent à un emploi généralisé d'engobes rouges, noirs et blancs. Des coupes, des bassins, des jarres et des vases à liquide à pâte rouge sont ornés de bandes et de taches peintes à l'engobe orange ou rouge et sont souvent associés à des objets de mêmes fonctions réalisés en pâte grise, engobés en noir. Par ailleurs, quelques cruches sont entièrement enduites d'un engobe blanc. En l'état des

35 Des analyses ont permis d'attribuer à Damas une production de vaisselle sur la base d'un raté de cuisson trouvé dans un cimetière de la banlieue de la ville. La composition de la pâte d'un carreau de style ottoman d'Iznik, conservé au Metropolitan Museum of Art de New York, atteste l'existence de cette production damascène à l'époque ottomane. M. JEnkins, «Mamluk Underglaze-Painted Pottery: Foundations for Future Study», Muqarnas, 2, 1984, p. 104, pl. 10 a.

${ }^{36}$ De 35 à $40 \%$ dans les contextes du début de la période et de 60 à $80 \%$ aux XVIII et $\mathrm{XIX}^{\mathrm{e}}$ siècles. Entre la fin du XV $\mathrm{XV}^{\mathrm{e}}$ siècle et le début du XVII ${ }^{\mathrm{e}}$, l'Unglazed Table Ware est représentée à hauteur de 15 à $20 \%$; la Plain Glazed Ware, de 25 à $35 \%$; la Coarse Unglazed Ware à $50 \%$. 
recherches, ce matériel semble caractéristique des niveaux du XVIII siècle et constitue la plus grande part de la vaisselle commune utilisée à cette époque. Comme sur de nombreux sites de Méditerranée ${ }^{37}$, on trouve aussi en abondance, pour le XVIII ${ }^{\mathrm{e}}$ et le début du $\mathrm{XIX}^{\mathrm{e}}$ siècle, des vases à filtre à pâte grise fine, poreuse, à une anse, ornés de décors géométriques tracés au peigne. Sans doute fabriqués à Damas, ils s'apparentent à certaines productions de même époque réalisées en Egypte ${ }^{38}$ et en Tunisie, à Djerba ${ }^{39}$.

L'absence de Hand Made Geometric Painted Ware, une céramique modelée dans une pâte grossière, peinte à l'engobe rouge de motifs géométriques, confirme bien le caractère très rural de cette production qu'on ne retrouve pas dans les fouilles de la forteresse de Damas, grand site urbain s'il en est.

Les marmites en terre ont complètement disparu sans doute au profit de chaudrons de cuivre étamé ${ }^{40}$. Les chandeliers de terre reprennent les formes de leurs homologues en métal — un socle tronconique ou parfois évasé est surmonté d'une collerette de laquelle s'échappe une tige creuse, souvent baguée, terminée en bulbe. Cette forme, réalisée en pâte rouge comme en pâte grise, est courante à partir du milieu du $\mathrm{XVI}^{\mathrm{e}}$ siècle. Enfin, comme partout sur les sites du Bilâd al-Châm, les chibouks ou pipes de terre sont nombreuses à la citadelle et caractérisent invariablement les niveaux d'occupations entre le XVII ${ }^{\mathrm{e}}$ et le $\mathrm{XIX}^{\mathrm{e}}$ siècle.

En l'état de la recherche, il est encore difficile de dater la vaisselle à pâte rouge, à glaçure plombifère monochrome sur engobe, mais on remarque toutefois qu'au point de vue morphologique elle n'entretient pas d'analogies avec les vases de même nature réalisés à l'époque mamelouke. Contrairement à ce qui a été parfois écrit, on ne note pas à la citadelle de Damas, de véritable continuité entre les productions mameloukes tardives et les fabrications ottomanes à l'exception de quelques

37 Voir par exemple pour Chypre et la Crète, M.L. von WARTBURG, 2001, op. cit., p. 382 ; M. HAHN, 1997, op. cit., p. 189-190; pour Istanbul, J.W. HAYES, 1992, op. cit., p. $275-276,300$.

38 A. RABAN, «The Shipwreck off Sharm-el-Sheikh», Archaeology, avril 1971, vol. 24, no 2, p. 146-155; H. Amouric, Fl. RicheZ, L. VAllauri, Vingt mille pots sous les mers, Aix-en-Provence, 1999, p. 173, 175.

39 J.W. HAYES, 1992, op. cit., p. 275-276, 300.

$40 \mathrm{~V}$. BIKIC signale le même phénomène à la forteresse de Belgrade, en revanche, les marmites de terre semblent nombreuses parmi les découvertes faites dans les fouilles de La Canée. 
types de vaisselle fine à pâte siliceuse. Ainsi la céramique peinte en noir sous glaçure alcaline bleu turquoise, très faiblement représentée dans les niveaux ottomans, semble être, du point de vue de la technique, une survivance des productions de même nature d'époque mamelouke. Cependant le décor, notamment composé d'œillets et de tulipes, est caractéristique du répertoire floral ottoman. À l'inverse, certains plats, sans doute datés du $\mathrm{XVI}^{\mathrm{e}}$ siècle, sont ornés de rinceaux végétaux peints en bleu sous glaçure alcaline incolore dans la grande tradition des fabrications mameloukes dont la décoration s'inspire des «bleu et blanc» chinois. Mais cette fois c'est la forme qui les distingue des exemplaires mamelouks. Il s'agit de très grands plats de service (de $40 \mathrm{~cm}$ de diamètre à l'ouverture), peu profonds, à marli chantourné et base annulaire très plate qui rappellent les plats qualifiés de boylu «très grand» dans les tarifs de prix évoquant les productions d'Iznik ${ }^{41}$. C'est la vaisselle fine à pâte siliceuse, peinte polychrome sous glaçure alcaline incolore, qui s'inspire pour une grande part des productions ottomanes anatoliennes d'Iznik, et sans doute issue d'ateliers damascènes, qui constitue la plus grande part de la vaisselle fine à pâte siliceuse de la citadelle. Elle peut être attribuée aux $\mathrm{XVII}^{\mathrm{e}}$ et $\mathrm{XVIII}^{\mathrm{e}}$ siècles. Il semblerait qu'ensuite les productions à pâte siliceuse disparaissent au profit de la vaisselle de table à pâte argileuse.

\section{3. «Importations» de vaisselle de table entre le XVI et la fin du XIX siècle}

Compte tenu du très faible volume des céramiques d'origines étrangères découvertes à la citadelle, il est difficile de parler d'importation un terme qui sous-entend un commerce organisé et un flux important de marchandises - cependant la présence de vaisselle d'origines très variées atteste la circulation de produits sur de très longues distances et leur commercialisation sur les marchés de la ville où l'on trouve tout à la fois des productions haut de gamme et des vases dont la rusticité n'a pas nui à une large distribution dans toute la Méditerranée.

Pour le $\mathrm{XVI}^{\mathrm{e}}$ siècle, on recense en très petites quantités : - des fragments de plat et de cruche d'Iznik, des productions de luxe également en usage dans les citadelles d'Alep et de Jérusalem ${ }^{42} ;-$ des fragments de

${ }^{41}$ N. Atasoy, J. Raby, 1990, op. cit., p. 43-44.

${ }^{42}$ N. Atasoy, J. RabY, 1990, op. cit., p. 98; C.N. Johns, 1950, op. cit., p. 190, pl. LXIII, 10. 
faïences «Bleu sur bleu» de Ligurie, de type calligrafico a volute tipo $c$, de la deuxième moitié du $\mathrm{XVI}^{\mathrm{e}}$ siècle et leurs imitations d'Italie du Nord ; - de la majolique toscane de Montelupo; - et quelques porcelaines «bleu et blanc» de Chine. D'autres productions italiennes sont attestées dans la seconde moitié du XVI ${ }^{e}$-première moitié du XVII ${ }^{\mathrm{e}}$ siècle, il s'agit de céramiques à décor d'engobes dite marmorizzate de Pise, et au XVII ${ }^{e}$ siècle de «Bleu sur Bleu » ligure d'Albisola à décor de palmettes. Ces vases côtoient des fincan-s de Kütahya, réalisés dans la première moitié du XVIII' siècle. C'est à Jérusalem que les découvertes de céramiques de Kütahya sont numériquement les plus importantes, tandis que les sites d'Acre, d'Antioche, de Burj al-Ahmar et de Tell Jezreel en ont livré quelques exemplaires seulement ${ }^{43}$. Une grande part des porcelaines de Chine date de la dynastie Qing (1644-1912), les types les plus fréquemment attestés dans les fouilles de Damas sont des coupelles et des tasses sans anse de Kraak porselein caractéristiques du style Wanli, de la fin XVI ${ }^{e}$-début XVII ${ }^{e}$ siècle ${ }^{44}$.

Au XVIII ${ }^{\mathrm{e}}$ siècle, la variété des importations n'est pas moins grande. On trouve des productions anatoliennes et thraces de Çanakkale et de Didymotique qui ont connu une très grande diffusion à travers l'ensemble de la Méditerranée mais au Bilâd al-Châm, en l'état des connaissances, Acre est le seul site ayant livré des vases issus de ces centres ${ }^{45}$. Les fabrications italiennes sont toujours présentes mais cette fois sous la forme de quelques écuelles «à taches noires» d'Albisola seulement attestées à $\mathrm{Acre}^{46}$. Les porcelaines européennes de Meissen et de Vienne, groupées sous le nom générique de porcelaines de Saxe, atteignent le Proche-Orient dès le deuxième quart du XVIII ${ }^{\mathrm{e}}$ siècle. Très appréciées dans l'ensemble de l'Empire ottoman, un nombre considérable de pièces y est importé ${ }^{77}$ mais seules deux tasses ont été trouvées à la citadelle. De

43 J. Carswell, 1972, op. cit.; G. Edelstein, M. AvisSar, "A Sounding in Old Acre», 'Atiqot, XXXI, 1997, p. 133, pl. III, 2-4; F. WAAGE, Antioch-on-the-Orontes, IV, Ceramics and Islamic Coins, Princeton, 1948, fig. 87, 6, 7; D. PRINGLE, The Red Tower (al-Burj al-Ahmar): Settlement in the Plain of Sharon at the Time of the Crusaders and Mamluks, AD 1099-1516, British School of Archaeology in Jerusalem Monograph Series, 1, Londres, 1986, p. 157-158, fig. 51, no $88-90$; A. GREY, «Pottery of the Later Periods from Tell Jezreel: an Interim Report», Levant, XXVI, 1994, p. 60.

${ }^{44}$ Elle doit son nom aux caraques portugaises qui transportèrent pour la première fois cette porcelaine en Occident à la la fin du XVI e siècle. M. L'Hour, L. LONG, E. RIETH, Le Mauritius, Grenoble, Casterman, 1989, p. 146.

${ }^{45}$ G. Edelstein, M. Avissar, 1997, op . cit., p. 132, fig. $1, \mathrm{n}^{\circ} 9$.

46 Ibidem, p. 131, nº11, mais non identifiées comme céramiques d'Albisola par les auteurs.

${ }^{47} \mathrm{~J}$. Soustiel rapporte la présence à Meissen d'un négociant turc qui, dans la première 
tels objets ont aussi été découverts à Acre et à Jérusalem ${ }^{48}$. D'élégantes tasses de type Dead-Leaf Brown couvertes d'une glaçure opaque colorée aux oxydes de fer, une production populaire au XVIII ${ }^{\mathrm{e}}$ siècle en Chine, et d'exceptionnelles coupelles monochromes dites «jaune impérial» ainsi que des porcelaines imari chinoises au décor peint en bleu combiné à des émaux rouges et verts représentent les principaux types venus de Chine. Mais tous ces objets de grands prix restent rares dans la fouille.

Les faïences anglaises polychromes qui proviennent des grandes fabriques industrielles du Staffordshire sont encore trop rarement considérées comme du matériel archéologique et identifiées comme tel. Cependant, pour qui sait les reconnaître, il est intéressant de constater combien elles ont circulé à travers le monde ${ }^{49}$. Des coupelles peintes aux couleurs vives issues des manufactures anglaises sont en usage à la citadelle dans la deuxième moitié du XIX ${ }^{\mathrm{e}}$ siècle. De grands plats peints en bleu de décors géométriques couvrants issus de la Fabrique impériale et royale de Nimy, près de Mons en Belgique, datent également de cette période tardive. Ils côtoient une assiette en faïence venue des ateliers de Moustier en Provence, de grands plats au décor floral en camaïeu de bleu imprimé du modèle «Flora» des fabriques de Creil-Montereau et des assiettes creuses à marli, en porcelaine blanche, fabriquées autour de 1875 à Sarreguemines en Moselle. Ces vases constituent les importations les plus récentes mises au jour à la citadelle.

Au-delà des typologies nécessaires à toutes recherches céramologiques et utiles pour les calages chronologiques sur les chantiers de fouilles, à l'issue de cette étude qui, je le rappelle, est en cours, nous obtiendrons une meilleure image des ustensiles de stockage, de cuisine, et de service employés par les janissaires mais aussi par les habitants «civils» qui résidaient dans cette «ville-garnison» qu'était devenue la citadelle de Damas. Cette recherche livrera aussi un panorama de la vaisselle disponible sur les marchés de la capitale d'une des provinces arabes de l'Empire.

moitié du XVIII ${ }^{e}$ siècle, passe commande de deux mille petites tasses à café «dans le goût turc », puis signe avec la manufacture un contrat au terme duquel, en échange de l'exclusivité, il s'engage à acheter chaque année la même quantité de tasses. J. SoustiEL, La céramique islamique, Fribourg, 1985, p. 348-349.

48 G. Edelstein, M. Avissar, 1997, op. cit., p. 133, pl. IV, 5 a et b; J. Carswell, 1972, op. cit., fig. 32 .

${ }^{49}$ H. AMOuRIC et al., 1999, op. cit., p. 177-180. 


\section{CROISEMENT DES SOURCES ÉCRITES ET DES ARTEFACTS :}

UNE APPROCHE À DÉVELOPPER

Croiser les données textuelles avec les données matérielles est une démarche jusqu'alors peu pratiquée par les archéologues et par les historiens ottomanisants. Pourtant ce double éclairage devrait enrichir notre connaissance de la culture matérielle, les objets donnant corps aux textes qui les évoquent et les textes donnant vie aux artefacts en les nommant, en précisant leur fonction et en les replaçant dans leur contexte d'utilisation. Les archives ottomanes, qu'il s'agisse de livres de comptes et des inventaires des cuisines impériales, des textes relatifs au trésor du palais, de registres de successions, de ventes, de confiscations, de tarifs des prix, d'inventaires après décès, fournissent des renseignements de tous ordres sur la vaisselle. Ces documents, souvent compilés par des fonctionnaires et des administrateurs, concernent en majorité le palais impérial à Istanbul ou les palais de province et la haute société ${ }^{50}$, plus rarement l'homme «ordinaire» mais on y traite aussi bien de la vaisselle de luxe et de service, que des pots and pans utilisés en cuisine, que des jarres de stockage et des écuelles destinées aux repas des serviteurs et des janissaires. Pour leur part, les découvertes archéologiques concernent à peu près tous les milieux et offrent un panorama assez vaste des objets employés pour le transport des denrées, leur stockage, la préparation des mets et leur service.

Toutes les périodes historiques n'offrent pas l'opportunité de confronter ces données les unes aux autres, c'est pourquoi il serait dommage de se priver des indications - éparses mais nombreuses - que les importantes collections d'archives ottomanes peuvent livrer. À titre d'exemples, voyons, à travers quelques sources écrites, quels types de renseignements il est possible d'obtenir sur la vaisselle.

\section{Nommer la vaisselle et préciser sa fonction}

Les fouilles livrent des artefacts de terre cuite muets que nous désignons par des termes céramologiques qui ne rendent pas toujours

50 Voir par exemple, les livres de comptes du palais de Mehmed à Manisa qui recensent les dépenses faites, entre 1594 et 1595 , pour le transport, le stockage de la nourriture et la préparation des repas. À cette occasion, sont énumérés de nombreux ustensiles dont la matière et la fonction sont précisées. F.M. EMECEN, «The Şehzade's Kitchen and its Expenditures an Account Book from Şehzade Mehmed's Palace in Manisa, 1594-1595 », in S. Faroqhi, C.K. Neumann (eds.), The Illuminated Table, the Prosperous House, Beiruter Texte und Studien 73, Würzburg, 2003, p. 89-103. 
compte de façon précise de leurs fonctions. Les textes, quant à eux, fournissent des listes de termes dont la réalité est parfois difficile à appréhender parce que le turc est mélangé à l'arabe et au persan — «si les mêmes termes peuvent désigner des objets différents, des termes différents s'appliquent évidemment à désigner les mêmes biens: ces actes posent des problèmes de traduction liés à l'hétérogénéité linguistique de l'Empire ${ }^{51}$ », et parce qu'il est d'autant plus difficile aux historiens de donner un sens à ces mots qu'ils ignorent tout de la réalité qu'ils recouvrent. Cependant un va-et-vient plus systématique entre textes et objets pourrait améliorer les identifications.

Parfois des inscriptions directement peintes sur les plats désignent les mets qu'ils sont destinés à contenir. Ainsi dans la collection de porcelaines chinoises de Topkapı Sarayı, on lit, tracée à l'encre sous la base d'une coupe de «bleu et blanc», une inscription en arabe évoquant le kebab de poulet et, sous une autre, le mot narenciye qui fait référence aux oranges ou aux citrons ${ }^{52}$. Dans ces deux cas, il n'y pas d'ambiguïté quant à l'utilisation des plats mais il n'en est pas toujours de même comme le montre N. Atasoy lors de la mise en corrélation des objets et des définitions d'usage de vases recueillis dans différents types de textes relatifs aux productions d'Iznik ${ }^{53}$. Ces archives nous apprennent l'existence de kavanos-ı mâst (jarre à condiment), de kavanos-i tursi (jarre à yaourt); de kâse-i-hoşaf ou compotier, un récipient profond à large ouverture; de şerbet kâsesi (bol à sirop); de tabak-i sükker ou plat à confiserie; de kaymak tabağl (plat pour la crème caillée); de tabak-i gülbeşeker (plat à confiture de pétales de roses); de kuzu tabağ pour l'agneau); de tabak-i pâlûde ou helva tabağg (plat à dessert); de selita (plat à salade); de fincan (tasse à café). Malgré ces précisions et en l'absence de description plus fine, il n'est pas toujours aisé de rapprocher les plats existants des termes utilisés. C'est pourtant à une tâche semblable que je me suis employée en confrontant les inventaires après décès de Damascènes et de pèlerins morts à Damas au XVIII ${ }^{e}$ siècle des documents étudiés par C. Establet et J.P. Pascual ${ }^{54}$ — et les décou-

51 C. Establet, «Les intérieurs damascains au début du XVIII ${ }^{\mathrm{e}}$ siècle...sous bénéfice d'inventaire », in D. Panzac (dir.), Les villes dans l'Empire ottoman : activités et sociétés, II, Paris, CNRS Editions, 1994, p. 33.

52 N. ERBAHAR, «Non Chinese Marks and Inscriptions », in Chinese Ceramics in the Topkapi Saray Museum I, Londres, 1986, p. 125-138.

53 N. AtASOY, 1990, op. cit., p. 37-49.

54 C. Establet, 1994, op. cit.; J.P. Pascual, C. Establet, Familles et fortunes, 450 foyers damascains en 1700, IFEAD, 1994; C. Establet, J.P. PAscuAL, Ultime voyage 
vertes matérielles faites à la citadelle de Damas. Pour la préparation des aliments, on trouve dans les inventaires après décès la mention de bassins dits legen qui pourraient correspondre aux grands bassins à pâte rouge trouvés dans la fouille; des marmites et des casseroles appelées tangara, de grosses marmites dites ma'un ainsi que des poêlons appelés tawa, luhûq ou miqlâya sont utilisés pour la cuisson, ces objets sont sans doute en métal car, comme nous l'avons signalé, les marmites et les lèchefrites de terre sont absentes des fouilles de la citadelle. L'eau est servie dans des aiguières ou ibrik très présentes sous diverses formes parmi nos découvertes; dans des cruches dites batiya qu'on pourrait identifier avec des vases de plus petites tailles à pâte rouge; et dans des barradiyya pour lesquels il est précisé que ce sont des vases en terre poreuse qui servent à rafraîchir l'eau — une description qui s'applique tout à fait aux cruches à filtre, à pâte grise, au décor peigné, trouvées en grand nombre à la citadelle. Des gobelets appelés hasa sont utilisés pour boire mais nous n'en avons pas découvert d'exemplaire. Pour la conservation, on trouve, dans les inventaires, des pots à beurre en terre vernie dits dabbiyya mais, en l'absence de précision morphologique supplémentaire, il est difficile d'attribuer cette fonction aux nombreux pots glaçurés, à lèvre en bandeau, trouvés à la citadelle. Enfin il existe diverses jarres de resserre nommées hawabi et martabân qui pourraient correspondre, à Damas, aux jarres de taille moyenne, à deux anses, à pâte grise ou rouge, ou aux très grosses jarres engobées ou partiellement glaçurées. Les mets sont présentés dans des plats - tabaq - parfois en faïence; ils sont consommés dans des écuelles en terre - zabdiyya dont nous possédons de nombreux exemplaires ou dans des assiettes en faïences dites sahn ainsi que dans des bols appelés mashraba. Pour certains plats, l'appellation qaşani indique qu'il s'agit de céramique fine à pâte siliceuse et glaçure alcaline en rapport avec les productions perses de Kashan d'où le qualificatif est dérivé. Les plats et les coupes de diverses tailles mis au jour à la citadelle se rapportent effectivement à cette fonction de service et ils sont de diverses natures. Dans les inventaires mobiliers, ce sont les fincan-s qui sont le plus fréquemment cités. Ces petites tasses à café, des godets hémisphériques plus ou moins évasés sans anse et toujours montés sur une base annulaire cylindrique, sont très répandues dans l'Empire. Si les inventaires précisent la qualité de ces tasses, les termes employés recouvrent une réalité parfois difficile à pour La Mecque. Les inventaires après décès de pèlerins morts à Damas vers 1700, Damas, IFEAD, 1998. 
appréhender. On y distingue par volume décroissant de simples fincan-s sans qualificatif particulier; viennent ensuite des tasses 'asali, une épithète mystérieuse qui évoque le miel et qui fait peut-être référence à la couleur du matériau ou de la glaçure; des tasses dites sini c'est-à-dire en faïence commune ${ }^{55}$; on trouve des tasses qualifiées de djadid; ou encore des tasses gümüslü, c'est-à-dire en argent ou argenté, ce terme, plutôt que de faire référence à un décor de lustre métallique, évoque peut-être les sertissages en métal destinés à protéger des chocs les parties sensibles de l'objet; quelques fincan-s sont dits qaşani, c'est-à-dire à la manière des productions perses de Kashan et enfin les tasses farfuri renvoient aux porcelaines de Chine. Comme en écho à ces descriptions, on trouve, à la citadelle de Damas, des tasses de diverses sortes. Là encore, les résultats sont mitigés. Plusieurs termes semblent s'appliquer au même usage sans qu'il soit possible de préciser les différences fonctionnelles qui distinguent tel pot de tel autre et l'on peut aussi associer à un même mot plusieurs objets.

\section{Cuisine ottomane et pratique de table: de leur impact sur la vaisselle}

Les miniatures sont aussi une source à exploiter pour préciser quelles sont les pratiques de table en usage à la cour impériale et par conséquent quels sont les plats et autres objets utilisés. Ainsi, par exemple, dans une scène de banquet du Surnâme de Murad III, daté de 1582, un plat individuel est placé devant chaque convive alors qu'au début du XVIII siècle, les banqueteurs, figurés dans le Surnâme d'Ahmed III, plongent leur cuillère dans un grand plat de service unique. Bien entendu la prudence s'impose lors de la lecture de ces images qui ne sont pas des instantanés pris sur le vif et qui obéissent sûrement à des conventions de représentation. Il n'empêche qu'elles constituent tout de même une source supplémentaire pour l'histoire de la vaisselle.

Les récits des voyageurs et les rapports des diplomates évoquent parfois les ustensiles employés au cours des repas et la façon de s'en servir. Si vers 1712, l'ambassadeur de Pologne est impressionné par la porcelaine de Chine et les grands plateaux d'argent utilisés au palais impérial pour servir les mets, il se plaint de l'absence de couteau, de fourchette et même d'assiette ${ }^{56}$.

55 Dans le monde ottoman, şini désigne d'abord la céramique venue de Chine puis, par extension, ce terme définit aussi les faïences bleues et blanches.

56 N. SAKAOĞLU, «Sources for our Ancient Culinary Culture», in S. Faroqhi et C.K. Neumann, 2003, p. 56. 
$\mathrm{Au} \mathrm{XIX}^{\mathrm{e}}$ siècle, l'adoption, à la cour du Sultan et dans la haute société, de la culture culinaire occidentale entraîne non seulement l'introduction de nouveaux produits, de recettes nouvelles mais aussi de pratiques de table différentes. On utilise alors une table plutôt qu'un plateau, on s'assoit plutôt sur une chaise que sur le sol et l'on emploie une fourchette plutôt que ses doigts pour porter les aliments à sa bouche. Mahmud II est le premier sultan à manger assis sur une chaise, à une table. Coexistent alors deux manières de table: alaturka et alafranca. Les registres de la cuisine impériale de cette époque précisent que la vaisselle, comme les assiettes, les coupes, les plats de service et les verres sont généralement importés d'Europe, une pratique qui a commencé au $\mathrm{XVIII}^{\mathrm{e}}$ siècle comme le révèlent les inventaires après décès de certains dignitaires ottomans ${ }^{57}$. Des pièces venues des fabriques de porcelaine de Meissen et de Vienne côtoient des services réalisés dans les manufactures de Vincennes en 1738, puis dans la manufacture royale de Sèvres à partir de 1805 et dans les fabriques de Dresde en 1825. Les textes en rapport avec les cuisines impériales montrent qu'au début du XIX ${ }^{\mathrm{e}}$ siècle, les porcelaines européennes (françaises, allemandes et anglaises) sont préférées aux porcelaines chinoises et aux productions turques elles-mêmes. Les découvertes archéologiques faites dans une grande partie de l'Empire témoignent bien de cet engouement pour la vaisselle venue d'Europe même si les quantités d'objets mises au jour sont faibles.

La confrontation des textes, des miniatures et des données matérielles ne permet sans doute pas d'identifier toutes les formes en fonction à l'époque ottomane ni d'attribuer un nom à chaque objet, cependant il s'agit d'une tentative qui nourrit l'étude du mobilier céramique et qui donne davantage de corps à la culture matérielle et aux pratiques de table en usage dans l'Empire ottoman. Ce sont des pistes de recherches à explorer.

Il reste encore beaucoup de chemin à parcourir avant de pouvoir traiter, au travers de l'étude de la vaisselle ottomane, de l'histoire économique et sociale de l'Empire. Cependant la multiplication des publications et, on peut l'espérer, des typologies nouvelles, devraient contribuer à

${ }^{57}$ F.M. GöCEK, Rise of the Bourgeoisie, Demise of Empire: Ottoman Westernization and Social Change, Oxford, New York, 1996. 
une meilleure connaissance des différents ustensiles de terre cuite employés par les habitants de l'Empire qu'ils soient modestes ou riches, citadins ou paysans. Si de telles recherches se développent, et il semble que ce soit le cas, à terme, il sera possible d'établir des comparaisons entres sites de façon, d'une part, à mettre en lumière des spécificités régionales et, d'autre part, à souligner des permanences dans les manières de table quelles que soient les provinces de l'Empire ottoman. 


\begin{abstract}
Annexe
Cet inventaire très complet a été établi par M. Milwrigh (Oriental Institute, Oxford).

Les tableaux suivants ainsi que la bibliographie ont été repris de M. Milwright, "Pottery of Bilad al-Sham in the Ottoman Period : a Review of the Published Archaeological Evidence", Levant 32, 2000, p. 189-208.
\end{abstract}

Table 1. Sites in Bilad al-Sham reporting diagnostic pottery of the Ottoman period

\begin{tabular}{|c|c|c|c|}
\hline & Site Name & Publications & Comments \\
\hline 1 & Abila & John Wineland, pers. comm. & Tobacco pipes (unpublished) \\
\hline \multirow[t]{6}{*}{2} & ${ }^{\mathrm{c} A k k a}$ (Acre) & Pringle 1984 & Sixteenth- to seventeenth-century Italian glazed wares $(40)$ \\
\hline & & Muqari 1996 & $\begin{array}{l}\text { Tobacco pipes, narghile, imported Turkish glazed wares } \\
\text { (fig.134:1-2), cooking pots, lamps, and storage jars }\end{array}$ \\
\hline & & Stern 1997 & Tobacco pipes and other pottery $(65-68$, figs 18,19$)$ \\
\hline & & Berman 1997 & Tobacco pipes (99). \\
\hline & & Edelstein and Avissar 1997 & $\begin{array}{l}\text { Tob\&ico pipes, Chinese, Turkish, and European imports, } \\
\text { and locally-produced wares (132-135, figs 1:7-14;2;3, } \\
\text { colour pls III:2-4; IV:5-6) }\end{array}$ \\
\hline & & Nir 1997 & $\begin{array}{l}\text { Pottery associated with the reconstruction of a Crusader wall } \\
\text { in the eighteenth or nineteenth century }(31-34) \text {. }\end{array}$ \\
\hline 3 & Aleppo & Bernus-Taylor 1981 & Tobacco pipes (484) \\
\hline 4 & Antioch & Waagé 1948 & Italian and Turkish imports $(91,99$, figs $87: 6-7 ; 88: 25-26)$ \\
\hline 5 & ${ }^{\mathrm{c} A y n}$ Bikura (nr. Sataf) & $\begin{array}{l}\text { Gibson, Ibbs and Kloner } \\
1991\end{array}$ & Tobacco pipes (fig. $24: 3-4$ ) \\
\hline 6 & ${ }^{\mathrm{c} A y n}$ Karim & Saller 1946 & $\begin{array}{l}\text { Tobacco pipes, unglazed lamps, and other modern unglazed } \\
\text { pottery }(178-180 \text {, pl. } 35: 1,8-15,17-19)\end{array}$ \\
\hline 7 & ${ }^{\mathrm{c} A y z a r i y y a(B e t h a n y)}$ & Saller 1957 & $\begin{array}{l}\text { Tobacco pipes, possible Italian sgraffiato, unglazed lamps, } \\
\text { and other unglazed pottery }(190,235,281,323-324, \text { no. } \\
178, \text { pl. 124:9) }\end{array}$ \\
\hline 8 & $\mathrm{Ba}^{\mathrm{c}}$ albak & Sarre 1925 & Tobacco pipes $(132$, figs $65,65 a)$ \\
\hline & Beirut & Turquety-Pariset 1982 & Tobacco pipes and narghile fragments (figs $4: 25 ; 5: 26-29$ ) \\
\hline & Beirut, Suq area & Anon. 1995 & $\begin{array}{l}\text { Tobacco pipes, 'Syrian polychrome,' and sixteenth- or } \\
\text { seventeenth-century porcelain (pls on } 16-17 \text { ) }\end{array}$ \\
\hline 10 & Belmont Castle & Simpson 1994 & Tobacco pipes $(14-15)$ \\
\hline 11 & Burj al-Ahmar & Pringle 1986 & $\begin{array}{l}\text { Tobacco pipes and 'Syrian polychrome' wares (figs } 34: 11,12 \text {; } \\
51: 88-90 \text { ). Tobacco pipes also reported in the survey (sites } 4 \text {, } \\
12,17 \text { ) }\end{array}$ \\
\hline \multirow[t]{3}{*}{12} & Damascus, Bab Sarija & Toueir 1973 & 'Syrian polychrome' ware (pl.IIIB:d-e) \\
\hline & $\begin{array}{l}\text { Damascus, Duma } \\
\text { district }\end{array}$ & Carswell 1972 & Chinese and Japanese ceramics \\
\hline & Damascus, al-Salihiyya & al- ${ }^{\circ}$ Ush 196 & Tobacco pipes (fig.6.27) \\
\hline 13 & $\begin{array}{l}\text { Damiyya al-Jadida, } \\
\text { Jordan valley }\end{array}$ & Kareem 1993 & Tobacco pipes ( $202-203$, fig. $89: 4)$ \\
\hline \multirow{4}{*}{15} & Dayr al-Kahf & Parker 1986 & Tobacco pipes (166, fig. 66:365) \\
\hline & Dhira $^{c}$ al-Khan & Kareem 1992 & Tobacco pipes (7) \\
\hline & & Kareem 1993 & Tobacco pipes (216-217, fig. $66: 6)$ \\
\hline & & Kareem 1997 & Tobacco pipes (368) \\
\hline 16 & Hama & Riis and Poulsen 1957 & $\begin{array}{l}\text { Tobacco pipes, 'Syrian polychrome,' fifteenth-century } \\
\text { Florentine maiolica, and fifteenth-century Spanish imports } \\
\text { (figs } 403-408,799,800,1069-1082 \text { ) }\end{array}$ \\
\hline & Hasban & Sauer 1994 & Tobacco pipes (273) \\
\hline 18 & Hawran & Meinecke-Berg 1983 & Italian sgraffiato (pl. 57:a) \\
\hline 19 & ${ }^{\mathrm{C}}$ Iraq al-Amir & Brown 1983 & Tobacco pipes (no.152) \\
\hline \multirow[t]{2}{*}{20} & Jarash & Tholbecq, forthcoming & Tobacco pipes (pl. XI:106-107) \\
\hline & & Clark et al. 1986 & Tobacco pipes (pl. XXVII:2) \\
\hline
\end{tabular}


Table 1. Sites in Bilad al-Sham reporting diagnostic pottery of the Ottoman period (continued)

Site Name

21 Jerusalem, 'Bet Eliyahu' Abu Raya 1997 Garden' Jerusalem, Citadel

Johns 1950

Jerusalem, Damascus

Gate

Jerusalem, Dung Gate Jerusalem, Ophel

Jerusalem, south side of Haram al-Sharif

22 Kabri/al-Tall

23 Karak

24 Karak Plateau

25 al-Khalil (Hebron)

26 Khirbat Faris

27 Khirbat Khamase

28 Khirbat Manawat

29 Khirbat Minya

30 Khirbat al-Nakhl

31 Khirbat Zikhrin

$32 \mathrm{Ma}^{\mathrm{c}}$ arrat al-Nu${ }^{\mathrm{c}}$ man

33 Madaba

34 Mayrun

$35 \mathrm{Mdaybi}^{\mathrm{C}}$

36 al-Mina

37 Nahal Minim, northern Negev

38 al-Nasira (Nazareth)

$39 \mathrm{Qal}^{\mathrm{c}}$ at al-Jac ${ }^{\mathrm{c}} \mathrm{bar}$

40 Qara Mazra ${ }^{\mathrm{a}}$ a

41 Ras al-Dabub

42 Ras al-Qabub

43 Rujm al-Hanu

44 Sataf

$45 \mathrm{Tal}^{\mathrm{c}}$ Afiq

46 Tal Arba in, Jordan Valley

47 Tal ${ }^{\mathrm{C}}$ Arqa

48 Tal Dutha (Dothan)

49 Tal al-Hasi

50 Tal Jazar

51 Tal Miqna

\section{Publications}

Bahat 1985

1926

Ben-Dov 1982 1994 Mader 1957

Stern 1998

Anon. 1983b

Mason 1995

Meyers 1981

1999 season

Lane 1938

1993

Bagatti 1984

Barkay 1986 1991

Kareem 1993

Sarkis 1988

Saller 1957

Bliss 1894

Toombs 1985

Gittlen 1993
Wightman 1989

MacAlister and Duncan

Kempinski and Neimeier

Milwright 1999a

Personal observation of Miller 1991 survey material

Jeremy Johns, pers. comm. Tobacco pipes and associated pottery (unpublished)

Amit 1991

Stepanski 1988-1989

$\mathrm{Mu}^{\circ}$ ta University Museum

Porter and Watson 1987;

Herr et al. 1991

Meyers, Strange and

Rosen and Goodfriend

Personal observation from surface deposits

Bernus-Taylor 1981

Banning et al. 1989

McGovern 1983

Gibson, Ibbs and Kloner

Kochavi 1977

Hakimian and Salamé-

MacAlister 1912

\section{Comments}

Tobacco pipes (102-103)

Italian sgraffiato (fig.45:21)

Italian sgraffiato, Spanish lustre, Turkish imports, and local glazed pottery (189-190, pl. LXIII:2-10)

Tobacco pipes, narghile fragments, and Italian sgraffiato

(pls 63; 67:7-8 [and see Pringle 1984])

Chinese pottery

Glazed tile fragments perhaps associated with the sixteenthcentury redecoration of the Dome of the Rock (199)

Ming porcelain, celadon, tobacco pipes, and possibly Turkish and Persian glazed wares $(372-373$, pls on $364-365,368$ )

Tobacco pipes, European porcelain, and 'Rushaya

al-Fukhkhar' wares (figs $22: 8-9 ; 23: 2,5,6$ )

Tobacco pipes, Chinese pottery, Italian sgraffiato, and

'Syrian polychrome' (II, cat. nos.33:5-11; 39:9, 11; 40:2; $46: 3 ; 61: 11-13)$

Tobacco pipes (site 435, unpublished)

(

Tobacco pipes (pl. 95)

Tobacco pipes (148-149)

Tobacco pipes (11)

Tobacco pipes and 'Rushaya al-Fukhkhar' ware (75)

Tobacco pipes (unpublished)

Tobacco pipes and other pottery (115)

'Syrian polychrome' and Turkish imports with dubious

provenance (1987, pls $12-13 ; 1995$, figs $4: d ; 5: a-c ; 6: c-e)$

Tobacco pipes (fig. $3: 29: 13$ )

Tobacco pipes (pl. 9:22:8-10)

Tobacco pipes (unpublished)

Italian maiolica and sgraffiato and Turkish imports

(Çanakkale style) (60-61, pl. XXII1.H, JJ)

'Gaza' ware

Sixteenth-seventeenth-century Italian marbled ware, sgraffiato ware, and maiolica (196-200, fig.69, pls 79-81 [see also Pringle 1984,40 , figs 4,5$]$ )

Tobacco pipes (unpublished)

Tobacco pipes (484)

Tobacco pipes and coffee cups (12)

Tobacco pipes (fig.5:14-15)

Tobacco pipes (figs $13,14: 4$; pls XXI:2; XXIV:2)

'Gaza' wares and modern porcelain (44-45, figs $21-23$ )

'Syrian polychrome' bowl (fig.15).

Tobacco pipes (185-186, fig.71:9)

Tobacco pipes (30, table XVI)

Tobacco pipes (323)

Tobacco pipes (122)

'Gaza' wares (106-108, pl.83)

Tobacco pipes (pl. 83:28)

'Gaza' wares (11) 


\section{4}

\section{VÉRONIQUE FRANÇOIS}

Table 1. Sites in Bilad al-Sham reporting diagnostic puttery of the Ottoman period (continued)

\section{Site Name}

52 Tal al-Mutasallim

53 Tal Qaymun (Yoqne ${ }^{c} a m$ )

54 Tal Saylun

55 Tal al-Shuna south (Nimrin)

56 Tantura (Dor)

Tantura maritime excavation

57 Tíinnik

58 Udhruh

59 Umm al-Jimal

$60 \mathrm{Zi}^{\mathrm{c}}$ rin (Tal Jezreel)

\section{Publications}

Schumacher 1908

Ben-Tor and Rosenthal

1978

Andersen 1985

Kareem 1993

Stern 1994

Gibson et al. 1999

Kingsley and Raveh 1996

Ziadeh 1995

Killick 1983

Parker in de Vries 1998

Grey 1994

\section{Comments}

Tobacco pipes (figs 279,280 e)

Tobacco pipes (fig.6:1-2).

Tobacco pipes (pl.15:276)

Tobacco pipes (203-204)

Tobacco pipes (fig.231)

Tobacco pipes and other pottery (106)

Tobacco pipes (pl.85)

Tobacco pipes, modern porcelain and other pottery (figs 1 ; L. $296 ; 16: 7-11,13)$

Tobacco pipes (fig. 13:2-4)

Hand-made wares found in a late-nineteenth- to earlytwentieth-century context (215-218)

Tobacco pipes, Turkish coffee cups, Chinese pottery, and 'Gaza' wares (60-61)

Simpson 1994

Table 2. Sites in Bilad al-Sham with possible eriidence of pottery of the Ottoman period.

\section{Site Name}

a Baysan

b Karak Plateau

c Khirbat Duhala

d Safad

e Shawbak

f Tal Mevorakh

g Tabariyya (Tiberias)

h Tal Jemneh

i Tal al-Safiyya

j Tripoli

k Wu'cayra

\section{Publications}

Zori 1966

Brown 1992

Sari 1992

Damati 1988-1989

Brown 1988

Stern 1978

Anon. 1983a

Schaefer 1989

Bliss and MacAlister 1902 Salamé-Sarkis 1980

\section{Comments}

Possible Italian sgraffiato (pl.10.f)

Evolution of hand-made pottery allied to changes in settlement patterns through the late-Mamluk and Ottoman periods (chapter 11)

Final occupation level (figs. 8-9)

Ceramics associated with a late-Mamluk period destruction of the citadel (160)

Final occupation level (237-240)

Ceramic vessel found with child burial (pls 6.4, 5, 21)

'Ottoman' pottery found in association with the eighteenthcentury (?) sea walls

'Gray ware' pottery found on surface and upper levels (42-43, figs. $7 ; 8.5-7)$

Possible Italian sgraffiato (pl.65)

Possible Italian sgraffiato (pl.XIVII.9)

\section{Bibliography}

Abu Raya, R. (1997) Jerusalem, 'Bet Eliyah.' ESI XVI, 102-103.

Altun, A., Carswell, J. and Öney, G. (1991) Turkish Tiles and Ceramics. Sadberk Hanim Museum: Istanbul.

Amit, D. (1991) Khirbat Khamase. ESI X, 148-149.

Andersen, F. (1985) Shiloh: the Danish Excavations at Tall Sailun, Palestine in 1926, 1929, 1932 and 1963. II. The Remains from the Hellenistic and Mamluk Periods. National Museum of Denmark: Cop enhagen.

Anon. (1983a) Tiberias. ESI II, 103.

Anon. (1983b) Khirbet Zikhrin. ESI II, 114-115. 
Anon. (1995) Beirut: Excavations of the Souk Area. Urban Archaeology ' 94 . Pamphlet issued by the Ministry of Culture and Higher Educations and the Directorate General of Antiquities, Lebanon.

Ashton, L. (1932) Near Eastern Pottery. The Connoissiur XC, No. 373, 160-168.

Atasoy, N. and Raby, J. (1989) Iznik. The Pottery of Ottoman Turkey. London.

Bagatti, B. (1984) Gli Scavi di Nazaret. II. Dal secolo XII ad oggi. Studium Biblicum Franciscanum Collectio Maior No. XVII. Jerusalem.

Bahat, D. (1985) Jerusalem, Dung Gate. ESI IV, 54.

Banning, E. et al. (1989) Wadi Ziqlab Project, 1987. A Preliminary Report. $A D A \mathcal{J}$ XXXIII, 43--58.

Barkay, G. (1986) Ketef Hinnom. A Treasure facing ferusalem's Walls. The Israel Museum Catalogue No. 274 (Summer). Jerusalem.

Ben-Dov, M. (1982) In the Shadow of the Temple: The Discovery of Ancient ferusalem. Trans. I. Friedman. Jerusalem.

Ben-Tor, A. and Rosenthal, R. (1978) The First Season of Excavations at Tel Yoqne'am, 1978. Preliminary Report. IEf XXVIII, 57-82, pls 16-18.

Berman, A. (1997) Excavation of the Courthouse Site at 'Akko: A Siege-Trench of Bonaparte's Army in Areas $\mathrm{TB}$ and TC. 'Atiqot 31: 'Akko (Acre): Excavation Reports and Historical Studies, 91-103.

Bernus-Taylor, M. (1981) Islamic Glazed Pottery. Pp. 473-498 in J. Matthers (ed.) The River Qoueiq, Northern Syria, and its Catchment. Studies Arising from the Tell Rifa'at Survey, 1977-1979. Part ii. BAR International Series, No. 98 (ii).

Bliss, F. (1894) A Mound of Many Cities or Tel el Hesy Excavated. Committee of the Palestine Exploration Fund: London.

Bliss, F. and MacAlister, R. (1902) Excavations in Palestine during the Years, 1898-1900. Palestine Exploration Fund: London.

Bresenham, M. (1985) Descriptive and Experimental Study of Contemporary and Ancient Pottery Techniques at Busra. Berytus XXXIII, 89-101.

Brown, R. (1983) The 1976 ASOR Soundings. Pp. 105-132 in N. Lapp (ed) The Excavations at Araq elEmir. Volume 1. AASOR XLVII.

(1988) Late Islamic Shobak: A Summary Report of the 1986. Excavations. $A D A f$ XXXII, 225-245.

- (1992). Late Islamic Ceramic Production and Distribution in the Southern Levant: A Socio-economic and Political Interpretation. Unpublished Ph.D. thesis. State University of New York, Binghampton.

Carswell, J. (1970) Archaeology and the Study of Later Islamic Pottery. Pp. 63-65 in D.S. Richards (ed.) Islam and the Trade of Asia: A Colloquium. Oxford.

- (1972) China and the Near East: The Recent Discovery of Chinese Porcelain in Syria. Pp. 20-25 in W. Watson (ed) The Westward Influence of the Chinese Arts from the 14th to the 18th Century. Colloquies on the Art and Archaeology of Asia. Percival David Foundation, London.
Carswell, J. and Dowsett, C. (1972) Kütahya Tiles and Pottery from the Ammenian Cathedial of St. Fames, Ferusalem. 2 Vols. Oxford.

Clark, V. et al. (1986) The Jerash North Theatre: Architecture and Archaeology, 1982-1983. Pp. 205-302 in F. Zayadine (ed) ferash Archaeological Project, 1981-1983. Ammin.

Cohen, A. (1989) Economic Life in Ottoman ferusalem. Cambridge Studies in Islamic Civilization. Cambridge.

Crowfoot, G. (1932) Pots, Ancient and Modern. PEFQS, 179-187, pls. I-III.

Damati, A. (1988-1989) Safed Citadel. ESI VII-VIII, 159-160.

Edelstein, G. and Avissar, M. (1997) A Sounding in Old Acre. 'Atiqot 31: 'Akko (Acre): Excavation Reports and Historical Studies, 129-136.

Einsler, L. (1914) Das Töpferhandwerk bei den Bauernfrauen von Ramallah und Umgegend. $Z D P V$ XXXVII, 249-266, pls. XLV-XLVII.

Franken, H. and Kalsbeek, J. (1975). Potters of a Medicval Village in the fordan Valley. Excavations at Tell Deir 'Alla: $A^{L}$ Medieval Tell, Tell Abu Ghourdan, Fordan. Amsterdam.

Gatt, G. (1885) Industrielles aus Gaza. ZDPV VIII, 69-79.

Gibson, S., Ibbs, B. and Kloner, A. (1991) The Sataf Project of Landscape Archaeology in the Judean Hills: A Preliminary Report on Four Seasons of Survey and Excavation (1987-89). Levant XXIII, 29-54.

Gibson, S., Kingsley, S. and Clarke, J. (1999) Town and Country in the Southern Carmel: Report of the Landscape Archaeology Project at Dor. Levant XXXI, 71-121.

Gittlen, B. (1993) Tel Miqne-Ekron. Report of the 1984 Excavations. Field III SE. American Schools of Oriental Research and Israel Exploration Society.

Grant, E. and Wright, G. (1938-1939) Ain Shems Excavations (Palestine). Part IV. Pottery. Part V. Text. Biblical and Kindred Studies, Nos. 7, 8. Haverford, Pennsylvania.

Grey, A. (1994) Pottery of the Later Periods from Tell Jezreel: An Interim Report. Levant XXVI, 51-62.

Hakimian, S. and Salamé-Sarkis, H. (1988) Céramiques médiévales trouvées dans une citerne à Tell 'Arqa. Syria LXV, 1-64.

Hankey, V. (1968) Pottery-Making in Beit Shebab, Lebanon. PEQ 100, 27-32, pls. VII-XVII.

Hayes, J. (1980) Turkish Clay Pipes: A Provisional Typology. Pp. 3-10 in P. Davey (ed) The Archaeology of the Clay Tobacco Pipe No. 4. BAR International Series, No. 92.

- (1992) Excavations at Saraçhane in Istanbul. II. The Pottery. Princeton.

Herr, L. et al. (1991) Madaba Plains Project. The 1987 Season at Tell el-'Umein and Vicinity and Subsequent Studies. Madaba Plain Project II. Andrews University Press: Berrien Springs.

Herr, L. and Trenchard, W. (1996) Published Pottery of Palestine. American Schools of Oriental Research: Atlanta. 
Hütteroth, W. and Abdulfattah, K. (1977) Historical Geography of Palestine, Transjordan and Southern Syria in the late Sixteenth Century. Nuremburg.

Johns, C. (1950) The Citadel, Jerusalem. A Summary of Work since 1934. QDAP XIV, 121-190, pls. XVIII-XXVIII.

Johns, J. (1998) The Rise of Middle Islamic Hand-Made Geometrically-Painted Ware in Bilâd al-Shâm. Pp. 65-93 in R.-P. Gayraud (ed) Colloque international d'archéologie islamique. IFAO, Le Caire, 3-7 février 1993. Textes arabe et études islamiques 36 . Institut français d'archéologie islamiques.

Johns, J., McQuitty, A. and Falkner, R. (1989) The Fâris Project: Preliminary Report upon the 1986 and 1988 Seasons. Levant XXII, 63-95.

Kareem, J. (1992) Darb al-Quful in the Light of Archaeological and Historical Evidence. Newsletter of the Institute of Archaeology and Anthropology, Yarmouk University XIII, 3-8.

- (1993) Settlement Patterns in the fordan Valley in the Mid- to Late-Islamic Period. Unpublished Ph.D. thesis. Freien Universität, Berlin.

- (1997) The Site of Dhra ${ }^{c}$ al-Khan: A Main Caravanserai on Darb al-Quful. SHAF VI, 365-369.

Kawatoko, M. (1996) Al-Tur: A Port City Site on the Sinai Peninsula. The 12th Expedition in 1995 (Summary Report). Tokyo.

Kempinski, A. and Niemeier, W. (1994) Excavations at Kabri. Preliminary Report of the 1992-1993 Seasons, 7-8. Tel Aviv.

Killick, A. (1983) Udruh - The Frontier of an Empire: 1980 and 1981 Seasons, a Preliminary Report. Levant $\mathrm{XV}, 110-131$

Kingsley, S. and Raveh, K. (1996) The Ancient Harbour and Anchorage at Dor, Israel. Results of Underwater Surveys, 1976-1991. Dor Maritime Archaeology Project. BAR International Series, No. 626.

Kochavi, M. (1977) Aphek-Antipatris. Five Seasons of Excavation at Tel Aphek-Antipatris (1972-1976). Israel Exploration Society: Tel Aviv.

Kubiak, W. (1969) Overseas Pottery Trade of Medieval Alexandria as shown by recent Archaeological Discoveries. Folia Orientalia X, 5-30.

Lane, A. (1938) Medieval Finds at Al Mina in North Syria. Archaeologia LXXXVII, 19-78.

(1957a) Ottoman Pottery of Isnik. Ars Orientalis II, 247-281.

- (1957b) Later Islamic Pottery: Persia, Syria, Egypt, Turkey. London.

MacAlister, R. (1912) The Excavation of Gezer 1902-1905 and 1907-1909. 2 vols. Palestine Exploration Fund: London.

MacAlister, R. and Duncan, J. (1926) Excavations on the Hill of Ophel, Jerusalem 1923-1925. PEFA IV, 196-200, pls. XVII-XXII.

MacDonald, B. (1988) The Wadi el Hasa Archaeological Survey, 1979-1983. West-central fordan. Waterloo.

McGovern, P. (1983) Test Soundings of Archaeological and Resistivity Survey Results at Rujm al-Henu. $A D A \mathcal{F}$ XXVII, 105-142.
Mader, E. (1957) Mambre. Die Ergebnisse der Ausgrabungen im heiligen Bezirk Râmet el-Halil in Südpalästina, 1926-1928. 2 Vols. Freiburg.

Mason, R. (1995) Defining Syrian Stonepaste Ceramics: Petrographic Analysis of Pottery from $\mathrm{Ma}^{\mathrm{c}}$ arrat Nu'man. Pp. 1-18 in J. W. Allan (ed) Islamic Art in the Ashmolean Museum. Oxford Studies in Islamic Art X, Pt. 2. Oxford

Mayer, L. (1934) A Hoard of Mamluk Coins. QDAP III, 167-171, pl. LXVIII.

Megaw, A. (1939) Three Medieval Pit Groups from Nicosia. Reports of the Department of Antiquities, Cyprus 1937-1939, 145-168, 224-226.

Meinecke-Berg, V. (1983) Keramik des italienischen Renaissance im Nationalmuseum von Damaskus. Damaszener Mitteilungen I, 243-247, pl. 57.

Mershen, B. (1985) Recent Handmade Pottery from North Jordan. Berytus XXXIII, 75-87.

Meyers, E., Strange, J. and Meyers, C. (1981) Excavations at Ancient Meiron, Upper Galilee, Israel 1971-72, 1974-75, 1977. Meiron Excavation Project, Vol. 3. American Schools of Oriental Research.

Miller, J.M. (1991) Archaeological Survey of the Kerak Plateau: Conducted during 1978-1982 under the Direction of F. Maxwell Miller and Jack M. Pinkerton. American Schools of Oriental Research Archaeological Reports No. 1. Atlanta.

Milwright, M. (1999a) Trade and Patronage in Middle Islamic fordan. The Ceramics of Karak Castle. Unpublished Ph. D. thesis. 2 Vols. Oxford University. (1999b) Pottery in Written Sources of the AyyubidMamluk Period (ca. 1171-1517). BSOAS LXIII, No. 2, 504-518.

- (forthcoming) Gazetteer of Archaeological Sites in the Levant reporting Pottery of the Middle Islamic Period (ca. 1100-1600). Islamic Art V.

Muqari, A. (1996) 'Akko, the Old City. ESI XV, 124-125.

Nir, Y. (1997) A Turkish Well in Area TC. 'Atiqot 31, 105-107.

Parker, S.T. (1986). Romans and Saracens: A History of the Arabian Frontier. American Schools of Oriental Research Dissertation Series No. 6. Winona Lake, IN.

Petersen, A. (1994) Ottoman Terracotta Vaulting Tubes. Orient Express III, 89-91.

Poole, J. (1995) Italian Maiolica and Incised Slipware in the Fitzwilliam Museum, Cambridge. Cambridge.

Porter, V. and Watson, O. (1987) Tell Minis Wares. Pp. 175-248 in J.W. Allan and C. Roberts (eds) Syria and Iran: Three Studies in Medieval Ceramics. Oxford Studies in Islamic Art IV. Oxford.

Pringle, D. (1984) Italian Pottery from late Mamluk Jerusalem: Some Notes on late and Post-Medieval Italian Tradewares in the Levant. Atti XVII. Convegno internazionale della ceramica. Temi liberi, 37-44.

- (1986) The Red Tower (al-Burj al-Ahmar): Settlement in the Plain of Sharon at the Time of the Crusaders and Mamluks, AD 1099-1516. British School of Archaeology in Jerusalem Monograph Series No. 1. London. 
al-Qasimi, J. (1960). Dictionnaire des metiers damascaines. Z. al-Qasimi and K. al-Azem (eds) Le Monde d'OutreMer passé et présent. Deuxième série. Documents III. 2 Vols. Paris.

Raban, A. (1971) A Shipwreck off Sharm el-Sheikh. Archaeology XXIV, No. 2 (April), 146-155.

Riis, P. and Poulsen, V. (1957), Hama, fouilles et recherches de la fondation Carlsberg, 1931-1938. IV, 2. Les Verreries et poteries médiévales. Nationalmuseets Skrifter. Større Beretninger, No. 3. Copenhagen, 115-301.

Robinson, R. (1985) Tobacco Pipes of Corinth and the Athenian Agora. Hesperia LIV, 149-203, pls. 33-64.

Rogers, M. (1989) Domestic Life in Palestine. Repr. of 1862 edition. Kegan Paul International: London.

Rosen, S. and Goodfriend, G. (1993) An Early Date for Gaza Ware from the Northern Negev. PEQ CXXV, 142-147.

Rosen-Ayalon, M. (1995) Rural Life and Urban Economics in the Holy Land during the Ayyubid, Mamluk and Ottoman Periods. Pp. 512-523 in T.E. Levy (ed.) The Archaeology of Society in the Holy Land. London.

Saller, S. (1946) Discoveries at St. Fohn's, 'Ein Karim, 1941-1942. Studium Biblicum Franciscanum No. 3. Jerusalem.

(1957) Excavations at Bethany (1949-1953). Studium Biblicum Franciscanum No. 12. Jerusalem.

Salamé-Sarkis, H. (1980) Contribution à l'histoire de Tripoli et de sa region à l'époque dis croisades: problemes d'histoire, d'architecture et de céramique. Paris.

Sari, S. (1992) Dohaleh, a new Site in Northern Jordan. Liber Annuus XLII, 259-277.

Sarre, F. (1925) Keramik und andere Kleinfunde der islamischen Zeit von Baulbek. Berlin and Leipzig.

Sauer, J. (1973) Heshbon Pottery 1971: A Preliminary Report on the Pottery from the 1971 Excavations at Tell Heshban. Michigan.

- (1994) Pottery at Hesban and its Relationships to the History of Jordan. Pp. 225-282 in D. Merling and L. Geraty (eds) Hesban after 25 Years. Berrien Springs.

Schaefer, J. (1989) Archaeological Remains from the Medieval Islamic Occupation of the Northwest Negev Desert. BASOR CCLXXIV, 33-60.

Schölch, A. (1993) Palestine in Transformation, 1856-1882. Studies in Social, Economic, and Political Development. Trans. W. Young and M. Gerrity. Institute for Palestine Studies: Washington, DC.

Schumacher, G. (1908) Tell el-Mutesellim. Leipzig.

Simpson, St. J. (1990a) A Brief Introduction to Ottoman Clay Pipes. Society of Clay Pipe Research Newsletter XXVII (July), 6-10

- (1990b) Ottomari Clay Pipes from Jerusalem and the Levant: A Critical Review of the Published Evidence. Society of Clay Pipe Research Newsletter XXVIII (October), 6-14.

- (1991) Smoking in Central Asia. Society of Clay Pipe Research Newsletter XXXII (October), 16-22.

-_. (1993) Turkish Clay Pipes: A Review. Society of Clay Pipe Research Newsletter XXXIX (Summer), 17-23.
(1994) Near Eastern Pipe News. Society of Clay Pipe Research Newsletter XIIV (Autumn), 14-15.

(1995) An Ordeal with a Pipe: Changing Attitudes to Smoking in the Near East during the 17th-18th Centuries. Society of Clay Pipe Research Newsletter XIVII (Summer), 17-22.

Stepanski, Y. (1988-1989) Horvat Minya. ESI VII-VIII, 73-75.

Stern, Edna (1997) Excavation of the Courthouse Site at 'Akko: The Pottery of the Crusader and Ottoman Periods. 'Atiqot 31: 'Akko (Acre): Excavation Reports and Historical Studies, 35-70.

(1998) Horvat Manot. ESI XVIII,10-11.

Stem, Eliezer (1989-1990) 'Akko. ESI IX, 104-105.

Stern, Ephraim (1978) Excavations at Tel Mevorakh (1973-1976). Part One: From the Iron Age to the Roman Period. Qedem IX.

(1994) Dor: Ruler of the Seas. Twelve Years of Excavations at the Israelite - Phoenician Harbor Town on the Carmel Coast. Israel Exploration Society: Jerusalem.

Tatcher, A. (1998) 'A Akko. ESI XVIII, 12-13.

Tholbecq, L. (forthcoming) Une installation d'époque médiévale dans la sanctuaire de Zeus (Jordanie): la céramique.

Toombs, L. (1985) Tell el-Hesi: Modern Military Trenching and Muslim Cemetery in Field I, Strata I-II. The foint Archaeological Expedition to Tell el-Hesi. Vol. 2. K. O'Connell (ed). Waterloo, Ontario.

Toueir, K. (1973) Céramiques mameloukes à Damas. Bulletin d'études orientales. XXVI, 209-217.

Turquety-Pariset, F. (1982). Fouilles de la municipalité de Beyrouth (1977): les objets. Syria LIX, 27-76.

Tushingham, A.D. (1985) Excavations in ferusalem, 1961-1967. Vol. 1. Royal Ontario Museum, Toronto.

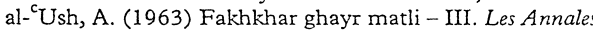
archéologiques arabes Syriennes XIII, 3-52 (Arabic section).

Vannini, G. and Tonghini, C. (1997) Medieval Petra Stratigraphic Evidence from Recent Archaeological Excavations at Wu'cayra. SHAJ VI, 371-384.

Vernoit, S. (1997) Occidentalism. Islamic Art in the Nineteenth Century. The Nasser D. Khalili Collection of Islamic Art No. 23. London.

de Vries, B. (1998) Umm el-fimal. A Frontier Town and its Landscape in Northern fordan. Volume 1. Fieldwork, 1972-1981. Journal of Roman Archaeology Supplementary Series No. 26. Portsmouth, Rhode Island.

Waagé, F. (1948) Antioch-on-the-Orontes. IV. Ceramics and Islamic Coins. Princeton.

Whitcomb, D. (1988) A Fatimid Residence at 'Agaba, Jordan. ADAf XXXII, 207-224.

Wightman, G. (1989) The Damascus Gate, Ferusalem: Excavations by C. $-M$. Bennett and 7. B. Henessy at the Damascus Gate, Ferusalem, 1964-66. BAR International Series No. 519.

Ziadeh, G. (1995) Ottoman Ceramics recovered from Ti innik, Palestine. Levant XXVII, 209-245.

Zori, N. (1966) The House of Kyrios Leontis at Beth Shean. IEf XVI, 123-134. 
Véronique FrANÇOIs, Tabak, ibrik, fincan et autres pots d'époque ottomane au Bilâd al-Châm

Des travaux épars et de natures diverses, écrits tant par des historiens de l'art que par des archéologues, ont livré des indications de toutes natures sur la vaisselle de terre fabriquée et commercialisée dans l'Empire ottoman. Cependant, les typologies de référence permettant de distinguer, par périodes chronologiques et par centres de fabrication, les céramiques qui répondent aux nécessités domestiques habituelles, c'est-à-dire la vaisselle culinaire, de service et de table et les céramiques de stockage et de transport, sont encore très rares comme le montre, dans cet article, l'inventaire des principales découvertes faites sur les territoires de la Sublime Porte. Dans ce contexte, le matériel ottoman mis au jour dans la fouille récente de la citadelle de Damas apporte des indications nouvelles sur la vaisselle en usage dans une des provinces arabes de l'Empire du $\mathrm{XVI}^{\mathrm{e}}$ au début du $\mathrm{XX}^{\mathrm{e}}$ siècle. L'étude de ces artefacts, combinée aux données fournies par les sources écrites, livre une image plus vivante des intérieurs damascènes et des pratiques de table en cours à l'époque ottomane.

Véronique FrançoIs, Tabak, ibrik, fincan, and other Pots dating from the Ottoman Period in Bilâd al-Châm

Scattered studies of various nature, written as well by art historians and by archaeologists, give all sorts of indications on earthenware, made and commercialized in the Ottoman Empire. However, typological references enabling us to distinguish by chronological periods and by fabrication centres, ceramics answering the usual domestic needs, that is to say cooking ustensils, service and table dishes, stocking and transport ceramics are still very rare as can be seen in the inventory of the chief discoveries made in the Sublime Porte territories published in this article. In this context, the Ottoman material discovered during the recent excavation of Damascus citadel brings new indications on the plates and dishes of use in one of the Arabian provinces of the Empire from the XVI ${ }^{\text {th }}$ Century to the beginning of the $\mathrm{XX}^{\text {th }}$ Century. The study of these artifacts, combined with the data furnished by written sources gives a livelier image of Damascene homes and table practices in the Ottoman period. 